\title{
The relationship between intracranial pressure and lactate/ /pyruvate ratio in patients with subarachnoid haemorrhage
}

\author{
Cesak T, Adamkov J, Habalova J, Poczos P, Kanta M, Bartos M, Hosszu T \\ Department of Neurosurgery, University Hospital Hradec Kralove, Charles University in Prague, \\ Faculty of Medicine in Hradec Kralove, Czech Republic. adamkov@seznam.cz
}

\section{ABSTRACT}

AIM: The aim of this study was to analyse the relationship between intracranial pressure (intracranial pressure monitoring) and lactate pyruvate ratio (cerebral microdialysis) in patients with ruptured intracranial aneurysms. METHODS: In a group of fifteen patients, intracranial pressure and lactate/pyruvate ratios were measured and logged in hourly intervals. The relationship between these two variables was subsequently analysed in two ways. 1) Intracranial hypertension (ICP $>20 \mathrm{mmHg}$ ) in the presence of energy deprivation (L/P ratio $>30$ ) was noted. 2) The dynamics of L/P ratio changes in relation to immediate ICP and CPP values was analysed.

RESULTS: Out of a total of 1873 monitored hours we were able to record lactate/pyruvate ratios higher than 30 in 832 hours (44\%). Of those 832 hours during which lactate/pyruvate ratios were higher than 30, ICP was higher than 20 in 193 hours (23\%). Out of 219 hours of monitoring, in which ICP was higher than 20, a simultaneously increased L/P ratio higher than 30 was recorded in 193 hours (88 \%). L/P ratio values above 30 were associated with decreased CPP values $(p=0.04)$, but not with increased ICP values $(p=0.79)$.

CONCLUSION: Intracranial hypertension coincides with energetic imbalance in approximately one quarter of cases. This points to the shortcomings of the most common form of neuromonitoring in SAH patients - ICP monitoring. This method may not be reliable enough in detecting hypoxic damage, which is the major cause of morbidity and mortality in SAH patients (Fig. 5, Ref. 11). Text in PDF www.elis.sk.

KEY WORDS: subarachnoid haemorrhage, intracranial pressure, brain microdialysis, lactate/pyruvate ratio.

\section{Introduction}

Despite reliable diagnostics, advanced neuromonitoring and therapy, subarachnoid haemorrhage due to ruptured intracranial aneurysms is still associated with a poor prognosis. Shortly after haemorrhage, approximately one third of all patients die. Another $45-50 \%$ die within 30 days of rupture (1). The treatment of ruptured intracranial aneurysms has become safer for the patient and the incidence of procedural complications has significantly decreased. The period following haemorrhage, during which patients are at risk of secondary cerebral injury is the major cause of high morbidity and mortality. These processes are the result of the way, in which brain cells and blood vessels react to blood and its breakdown products in the subarachnoid space with ensuing intracranial hypertension and cerebral hypoperfusion. Under such conditions, the energetic metabolism of brain cell is at stake (2).

Immediately after SAH there is a sharp increase in intracranial pressure (ICP), which may reach values between systolic and

Department of Neurosurgery, University Hospital Hradec Kralove, Charles University in Prague, Faculty of Medicine in Hradec Kralove, Czech Republic

Address for correspondence: J. Adamkov, MD, PhD, Department of Neurosurgery, University Hospital Hradec Kralove, Charles University in Prague, Faculty of Medicine in Hradec Kralove, Sokolska 5, CZ-500 05 Hradec Kralove, Czech Republic.

Phone: +420.776384367 diastolic blood pressure (3). Together with the thrombofibrinous clot at the point of rupture, intracranial hypertension helps in controlling haemorrhage (3). Although ICP may normalize to physiological values after several minutes in some patients, the resulting loss of vascular autoregulation and pathological processes at the microcirculation level may lead to imbalance between energy requirements and substrate availability (3). The standard of neuromonitoring in patients with $\mathrm{SAH}$ and altered consciousness is intracranial pressure monitoring. Current technological advances also enable the evaluation of cerebral energy metabolism using cerebral microdialysis. The relationship between the intracranial pressure values and the main indicator of metabolic stress (lactate : pyruvate ratio) may provide a better understanding of the cascade of events following SAH and also help to critically assess current methods of neuromonitoring in $\mathrm{SAH}$.

\section{Materials and methods}

During the period from February 2012 to December 2015, microdialysis and intracranial pressure sensors as part of multimodal monitoring were implanted in fifteen patients with subarachnoid haemorrhage. These were patients, in whom early extubation and clinical monitoring following aneurysm treatment was unlikely. In the case of symetrical blood distribution in the subarachnoid spaces on baseline CT, an intracranial pressure sensor and microdialyis catheter were implanted into the frontal lobe of the non- 


\section{9-142}

dominant hemisphere. In the case of blood distribution in the basal cisterns and Sylvian fissure, the catheter was implanted into the white matter of the frontal lobe ipsilateral to the dominant side of haemorrhage. Multimodal monitoring was initiated 1-3 days following SAH. In hourly intervals, microdialysate samples were taken and processed using Iscus analyser (CMA Microdialysis $\mathrm{AB}$, Sweden), which was present at the bedside. The interstitial cerebral lactate, pyruvate, lactate/pyruvate ratios, glucose and glycerol values were logged. To collect microdialysate samples, catheters with $10 \mathrm{~mm}$ semipermeable membranes with 20 and 100 $\mathrm{kDa}$ pores were used. The perfusion pump rate remained constant at $0.3 \mu \mathrm{l} /$ minute. Intracranial pressure was monitored using a Codman intracranial pressure sensor. Multimodal monitoring was initiated following the obliteration of the source of haemorrhage using surgical or endovascular techniques. In this series, six patients underwent surgical clipping and eight patients received endovascular treatment (coil or stent-assisted). In one patient, a combined approach was used.

Statistical analysis was done using R Development Core Team. R: A Language and Environment for Statistical Computing [Internet]. R Foundation for Statistical Computing; 2016. p. 3503. Available from: http://www.r-project.org).

In testing hypotheses, the first order probability error of less than 0.05 was considered to be the border of statistical significance. The distribution of the acquired data was analysed using the Anderson-Darling test. Normal distribution was not found, and the Spearman rank test was used to assess correlation.

\section{Results}

Out of a total of 1873 monitored hours, there were 832 hours, in which the lactate/pyruvate ratio was above 30 (44\%) (Fig. 1). Out of 832 hours of monitoring, in which the lactate/pyruvate ratio was above 30 , we were able to identify 193 cases, in which ICP was above $20 \mathrm{mmHg}$ (23\%) (Fig. 2). Out of 219 hours of monitoring, in which ICP was above $20 \mathrm{mmHg}$, simultaneously increased lactate/pyruvate ratio was present in 193 hours (88\%) (Fig. 3). Lactate/pyruvate ratios above 30 were associated with decreased cerebral perfusion pressure values $(p=0.04)$, but not with increased ICP values ( $p=0.79)$ (Fig. 5).

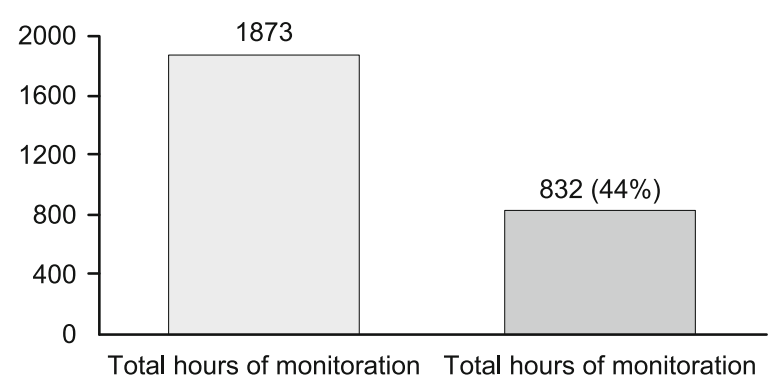

Fig. 1. Out of a total of 1873 monitored hours, an elevated lactate/ pyruvate ratio $>30$ was present in 832 hours, $44 \%$.

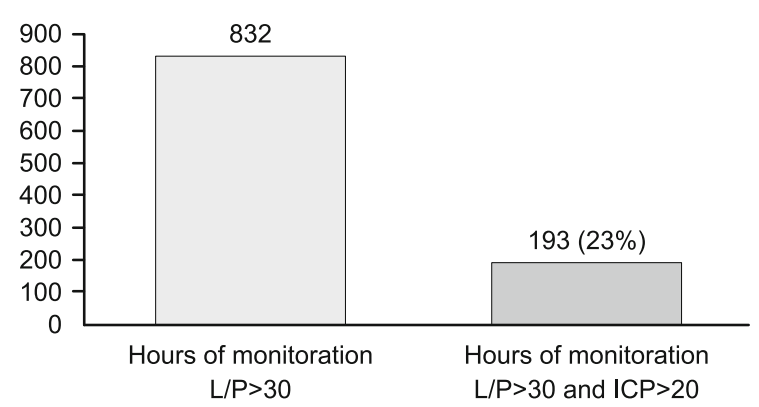

Fig. 2. Out of 832 hours of monitoring, in which the lactate/pyruvate ratio was above 30, we were able to identify 193 cases, in which ICP was above $20 \mathrm{mmHg}$ (23\%) (Fig. 2).

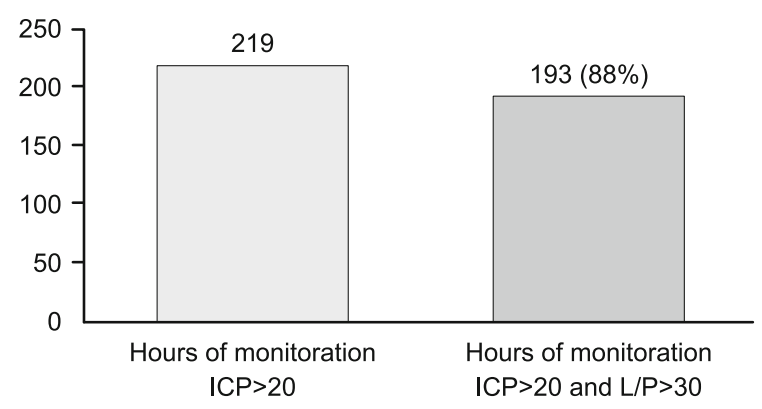

Fig. 3. Out of 219 hours of monitoring, in which ICP was above 20 mmHg, simultaneously increased lactate/pyruvate ratio was present in 193 hours (88 \%) (Fig. 3).

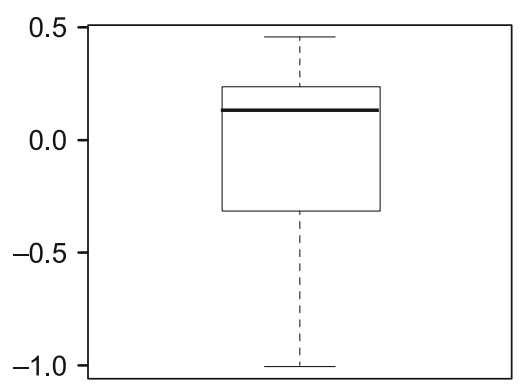

Fig. 4. A significant correlation was found in 4 out of a total of 15 patients. Overall, the correlation coefficients do not significantly differ from 0 (Fig. 4, $p=0.798$ ).

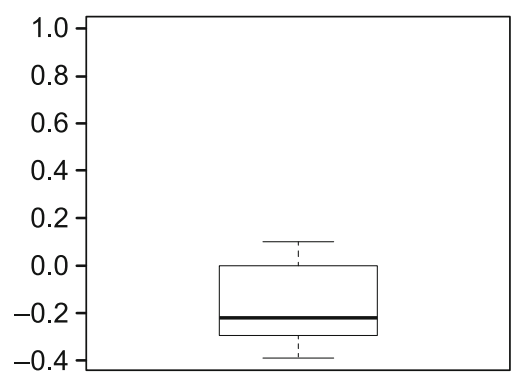

Fig. 5. A significant correlation was found in 4 patients out of a total of 15. Overall, the correlation coefficients do not significantly differ from 0 (Fig. 5, $p=0.043$ ). The median of individua correlations, Spear$\operatorname{man} r h o=-0.22$ shows that $\mathrm{raised} L / P$ ratio values are associated with lower CPP values. 


\section{Discussion}

Intracranial hypertension (ICH) following subarachnoid haemorrhage is a common phenomenon in patients in poor clinical condition, but also in patients with low Hunt-Hess scores (1-3). The most common cause of ICH is mass expanding intracranial hematoma, CSF flow obstruction or cerebral oedema. The increase in intracranial pressure is associated with a delayed neurological deterioration, changes in cerebral perfusion and elevated levels of excitotoxic mediators (4). Cerebral oedema is one of the causes of ICH following SAH and is a predictor of negative outcome (5). ICP monitoring remains the most common method of neuromonitoring of patients with severe brain injury. Cerebral perfusion pressure is determined as the difference between the mean arterial pressure (MAP) and ICP. Cerebral perfusion pressure determines cerebral blood flow and therefore an increase in ICP or a decrease in MAP may lead to cerebral ischemia. Several studies using jugular oximetry and monitoring of $\mathrm{PbtiO} 2$ in brain tissue demonstrated that cerebral ischemia may even develop under physiological ICP and CPP values (6). The fact that elevations in LP ratios in pericontusional cerebral tissue measured using microdialysis in patients with craniocerebral injury, does not correlate with changes in CPP, may explain why therapy based on correction of CPP in such patients is not always successful (7).

Chen et al studied the relationship between energetic crisis (L/P > 40), severe cerebral hypoxia (partial pressure of $\mathrm{O}_{2}$ in brain tissue, $\mathrm{PbtiO}_{2}<10 \mathrm{mmHg}$ ) and ICP in patients with severe $\mathrm{SAH}$. It was found that the sensitivity of ICP and CPP in detecting energetic crisis and severe hypoxia was only $21.2 \%$. In many cases, ICP and CPP values were within normal range, even when $\mathrm{L} / \mathrm{P}$ ratios and $\mathrm{PbtiO} 2$ were pathological (8).

In patients with intracranial hypertension following $\mathrm{SAH}$ in the first seven days after haemorrhage, increased L/P ratios were a marker of anaerobic metabolism. The result of which is cell membrane degradation and increased glycerol concentrations. SAH patients without ICP elevation exhibited significantly lower values of these parameters (9).

An interesting observation, which might be useful in clinical practice is that in $83 \%$ of cases a pathological elevation in microdialysis metabolites precedes ICP elevation by up to 6 hours (9). This study was also focused on the relationship between episodes of L/P ratios higher than 30 and raised ICP. Out of all the episodes of raised $\mathrm{L} / \mathrm{P}>30$, a simultaneous elevation in ICP above $20 \mathrm{mmHg}$ was present in only $23 \%$ (Fig. 2). Given energetic imbalance was present in only one quarter of episodes of increased intracranial pressure, this may be due to a variety of possible causes of increased $\mathrm{L} / \mathrm{P}$ ratios. This may arise due to processes affecting cerebral microcirculation. Hypoxia affects a generally small area of cerebral tissue. This may not be accompanied by an increase in intracranial pressure. There may be a disorder in vascular autoregulation leading to spasm or dilation and hyperemia of peripheral arterioles with thrombosis in the microcirculation. Another common cause of hypoperfusion leading to hypoxia and ischemia in peripheral cerebral tissue in the absence of ICH is cortical spreading depolarization (CSD). CSD is an extensive wave of depolarization of neuronal membranes in the cortex associated with an influx of cations and water intracellularly (10). The vascular response to the wave of depolarization is dependent upon the condition of cerebral tissue. Following SAH, vessels react by vasoconstriction resulting in a decrease in regional perfusion, which may lead to hypoxia and ischemia (10). Another possible cause of $\mathrm{L} / \mathrm{P}$ ratio elevation with unchanged ICP and even $\mathrm{PbtiO}_{2}$ is a dysfunction of oxygen utilization. In such cases, the cause may be due to mitochondrial dysfunction (high L/P ratio, low pyruvate, $\mathrm{PbtiO}_{2}$ unchanged) (11). The fact that intracranial pressure is elevated in only one quarter of patients of raised $\mathrm{L} / \mathrm{P}$ ratios suggests that multimodal monitoring is a necessity in this group of patients. Intracranial pressure monitoring alone is insufficient in identifying potential energetic failure early enough (hypoxia, mitochondrial dysfunction etc). An interesting observation is that 88 $\%$ of the patients with intracranial hypertension exhibit L/P ratios higher than 30 (Fig. 2). It is likely that intracranial hypertension is accompanied by a ,metabolic alarm“. In this study, the dynamics of $\mathrm{L} / \mathrm{P}$ ratios ( $>30)$ in relation to ICP and CPP were analysed. Based on the results, we can conclude that increases in $\mathrm{L} / \mathrm{P}$ ratio correlate more with a decrease in CPP than an increase in ICP $(\mathrm{p}=$ 0.043) (Figs 4 and 5). The conclusion is in agreement with Helbok et al, who described lower L/P ratios in increased CPP in their study (11). This is utilized in clinical practice by increasing CPP with catecholamines in cases of elevated $\mathrm{L} / \mathrm{P}$ ratios. The same study analysed ICP in patients with global cerebral oedema and without. Although patients with global cerebral oedema had significantly higher ICP values, these values did not reach $20 \mathrm{mmHg}$ (11). Therefore, ICP monitoring cannot reliably detect warning signs of metabolic dysfunction, which global cerebral oedema undoubtedly is.

\section{Conclusion}

Intracranial pressure monitoring is part of the standard care in SAH patients requiring artificial ventilation. Such patients are at risk of various complications such as: recurrent $\mathrm{SAH}$, cerebral oedema, vasospasm or cortical spreading depolarization. Some of these events involve elevated intracranial pressure, which makes ICP monitoring worthwhile. However, this is not always the case and ICP monitoring may be insufficient in detecting such cases. This study emphasizes the need for more detailed monitoring of patients in serious clinical condition following subarachnoid bleeding due to ruptured intracranial aneurysms. Microdialysis and analysis of cerebral energetic metabolites enables the early detection of potential secondary cerebral injury due to the cascade of processes following SAH. This enables detection of a redox change in neurons even in the absence of raised intracranial pressure. Such metabolic alarm precedes clinical manifestation so that therapeutic action can be taken as early as possible.

\section{References}

1. Feigin, V, Parag, V, Lawes, CM et al. Smoking and elevated blood pressure are the most important risk factors for subarachnoid hemorrhage in the Asia-pacific region: an overview of 26 cohorts involving 306,620 participants. Stroke 2005; 36: 1360. 
139-142

2. Adamkov J, Náhlovský J, Česák T et al. Relationship in between incoming clinical and graphical findings and early brain metabolic changes in patients after subarachnoid haemorrhage due to ruptured intracranial aneurysm. Cesk Slov Neurol N 2017; 80/113 (1): 75-79.

3. Grote E, Hassler W. The critical first minutes after subarachnoid hemorrhage. Neurosurgery 1988; 22: 654-661.

4. Sarrafzadeh AS, Thomale UW, Haux D, Unterberg AW. Cerebral metabolism and intracranial hypertension in high grade aneurysmal subarachnoid haemorrhage patients. Acta Neurochir Suppl 2005; 95: 89-92.

5. Hazell AS. Excitotoxic mechanisms in stroke: an update of concepts and treatment strategies. Neurochem Int 2007; 50: 941-953.

6. Stiefel MF, Udoetuk JD, Spiotta AM et al. Conventional neurocritical care and cerebral oxygenation after traumatic brain injury. J Neurosurg 2006; 105 (4): 568-575.

7. van Santbrink H, vd Brink WA, Steyerberg EW, Carmona Suazo JA, Avezaat CJ, Maas AI. Brain tissue oxygen response in severe traumatic brain injury. Acta Neurochir (Wien) 2003; 145 (6): 429-438.
8. Chen IJ, Stiefel M, Oddo M, Milby A, Maloney E, Frangos S, Levine J, Kofke W, LeRoux P. Detection of cerebral compromise with multimodality monitoring in patients with subarachnoid hemorrhage. Neurosurgery 2011; 69: 53-63.

9. Nagel A, Graetz D, Schink T, Frieler K, Sakowitz O, Vajkoczy P, Sarrafzadeh A. Relevance of intracranial hypertension for cerebral metabolism in aneurysmal subarachnoid hemorrhage. J Neurosurg 2009; 111 : 94-101.

10. Dreier JP, Woitzik J, Fabricius M, Bhatia R, Major S, Drenckhahn C, Lehmann TN, Sarrafzadeh A, Willumsen L, Hartings JA, Sakowitz OW, Seemann JH, Thieme A, Lauritzen M, Strong AJ. Delayed ischaemic neurological deficits after subarachnoid haemorrhage are associated with clusters of spreading depolarizations. Brain 2002; 129: 3224-3237.

11. Helbok R, Ko SB, Schmidt M et al. Global Cerebral Edema and Brain Metabolism After Subarachnoid Hemorrhage. Stroke 2011; 42: 1534-1539.

Received November 3, 2017. Accepted December 12, 2017. 